\title{
Detection of the PIK3CA Mutation in Circulating Tumor DNA as a Possible Predictive Indicator for Poor Prognosis of Early-Stage Breast Cancer
}

\author{
Ayaka Sato1,2, Masahiko Tanabe², Yumi Tsuboi' ${ }^{1}$, Masako Ikemura ${ }^{3}$, Keiichiro Tada ${ }^{2}$, \\ Yasuyuki Seto ${ }^{2}$, Yoshinori Murakami ${ }^{*}$ \\ ${ }^{1}$ Division of Molecular Pathology, Institute of Medical Science, The University of Tokyo, Tokyo, Japan \\ ${ }^{2}$ Department of Breast and Endocrine Surgery, Graduate School of Medicine, The University of Tokyo, Tokyo, Japan \\ ${ }^{3}$ Department of Pathology, Graduate School of Medicine, The University of Tokyo, Tokyo, Japan \\ Email: *ymurakam@ims.u-tokyo.ac.jp
}

How to cite this paper: Sato, A., Tanabe, M., Tsuboi, Y., Ikemura, M., Tada, K., Seto, Y. and Murakami, Y. (2018) Detection of the PIK3CA Mutation in Circulating Tumor DNA as a Possible Predictive Indicator for Poor Prognosis of Early-Stage Breast Cancer. Journal of Cancer Therapy, 9, 42-54. https://doi.org/10.4236/jct.2018.91006

Received: December 19, 2017

Accepted: January 23, 2018

Published: January 26, 2018

Copyright $\odot 2018$ by authors and Scientific Research Publishing Inc. This work is licensed under the Creative Commons Attribution International License (CC BY 4.0).

http://creativecommons.org/licenses/by/4.0/

(C) (i) Open Access

\begin{abstract}
Objectives: Circulating tumor DNA (ctDNA) is shown to provide the real-time genomic information of metastatic breast cancer. This study elucidates the clinico-pathological significance of ctDNA in early-stage breast cancer using the PIK3 CA mutation as an indicator. Materials and Methods: Twenty-seven primary breast cancers without metastasis were surgically resected and pathologically diagnosed at the University of Tokyo Hospital, Japan. Genomic DNA of primary tumor was extracted from formalin-fixed and paraffin-embedded specimens. ctDNA was extracted from fresh-frozen plasma from patients. The PIK3 CA mutations at E542K, E545K and H1047R were examined by Sanger sequencing or droplet digital PCR in 27 tumors and pre- and post-surgery plasma. Results: The PIK $3 C A$ mutations were detected in 13 (48\%) of 27 primary tumors. These mutations did not significantly correlate with specific clinico-pathological characteristics of tumors. When ctDNA was examined, 4 (33\%) of 12 cases carrying the mutated PIK $3 C A$ showed the identical mutation in pre-surgery plasma and $2(50 \%)$ of them showed the identical mutations in post-surgery plasma. Interestingly, in these 2 cases in pathological stages IIIA and IA, fractional abundance of the mutated PIK $3 C A$ alleles to the total alleles in pre-surgery ctDNA was around $1 \%$ or more and was higher than that of the other two cases without PIK $3 C A$ mutations in post-surgery ctDNA. Conclusions: The PIK $3 C A$ mutation in ctDNA is detectable even in a subset of early-stage breast cancer. Furthermore, fractional abundance of the mutated PIK $3 C A$ in pre-surgery ctDNA could provide a possible predictive indicator for tumor burden and for choosing the appropriate adjuvant treatment of breast cancer.
\end{abstract}




\section{Keywords}

Early-Stage Breast Cancer, PIK3CA, Circulating Tumor DNA, Plasma, Droplet Digital PCR

\section{Introduction}

Breast cancer is the most frequent malignancies in women in almost all the developed countries, including Japan. Whereas the most breast cancer cases surgically resected their tumors in early-stages, including pathological stages IA to IIIB, show favorably outcome, a subset of breast cancer showed recurrence with poor prognosis. Therefore, more accurate tumor markers for predicting the outcome of breast cancer are definitely required to overcome this type of cancer. Circulating tumor DNA (ctDNA) is a short fragment of tumor-derived DNA in the plasma, which is released into the peripheral blood from tumor cells mainly by apoptosis or necrosis [1]. Since the half-life of ctDNA is short, it may reflect the tumor burden of the patients in the real-time manner [2] [3]. Especially, when ctDNA carries a tumor-specific mutation, it would provide an ideal tumor biomarker which can be easily and repeatedly obtained through the plasma of patients. The technological advance in recent years enables us to quantify the ctDNA and to qualify its mutations [4]. Droplet digital PCR (ddPCR) is a new technique to quantify the number of target DNA molecules in the reaction tubes [5]. ddPCR is also highly sensitive to detect the rare mutated allele in the background of wild-type allele. Therefore, ddPCR is applicable to the detection of circulating tumor DNA if appropriate mutational targets are available for each type of tumors.

In breast cancer, in addition to the germline mutations of the BRCA1, BRCA2 and several related genes, number of somatic mutations are reported, including a tumor suppressor gene, TP53, and oncogenes, $A K T 1$ and PIK $3 C A$. Among them, 3 common mutations of the PIK $3 C A$ gene causing amino acid substitutions, H1047R, E545K and E542K, are the most frequent spots of mutation detected in breast cancer, especially in luminal type [6], and cover more than $80 \%$ of the PIK3CA mutations found in human cancer [7]. The PIK3CA gene encodes a p110 $\alpha$ catalytic subunit of phosphatidylinositol-3-kinase, which uses ATP to phosphorylate phosphatidylinositol (PtdIns), phosphatidylinositol-4-phosphate (PtdIns4P) and phosphatidylinositol-4, 5-biphosphate (PtdIns(4,5)P2), in cooperation with a p85 regulatory subunit. On the other hand, mutated proteins observed in cancer, including H1047R, E545K and E542K, activate AKT signaling to oncogenic growth of cancer cells. Previous study has reported that PIK $3 C A \mathrm{mu}-$ tations were detected in ctDNA from patients with metastatic breast cancer using digital PCR [8]. The levels of the mutated PIK3CA in ctDNA showed a larger dynamic range and more significant correlation with the tumor burden than other known tumor markers in metastatic breast cancer [9]. The PIK3CA muta- 
tion in ctDNA was also detected in the plasma from a subset of early-stage breast cancer patients, suggesting to provide a possible indicator of prognosis of the patients [10] [11] [12] [13].

In this prospective study, we examined the clinico-pathological significance of 3 common PIK $3 C A$ mutations in ctDNA from pre- and post-surgery plasma from 27 Japanese patients with early-stage breast cancer using ddPCR.

\section{Materials and Methods}

\subsection{Patients}

Twenty-seven Japanese female patients with primary breast cancer, who were intended to surgical resection at the University of Tokyo Hospital from January 2016 to September 2017, were enrolled in this prospective study. Patients with metastatic breast cancer or any additional tumors from different origins were excluded. Patients intended to neoadjuvant chemotherapy were also excluded from this study. We received written informed consent from all of the patients before pre-surgery blood collection. The genomic ethical review committee of University of Tokyo approved this study protocol (No. 27-01-0602) (Figure 1).

\subsection{DNA Extraction from Primary Tumors}

Specimens of primary tumors were obtained by core needle biopsy or surgical operation. Areas of the tumor were identified by pathologists on the basis of the histo-pathological examination of an adjacent section stained by hematoxylin and eosin. Genomic DNA was extracted from the unstained serially sectioned slides of the FFPE samples with QIAamp DNA FFPE Tissue Kit (Qiagen) according to the manufacturer's instructions.

\subsection{Blood Sample Collection and Extraction of ctDNA}

Peripheral blood samples of $10 \mathrm{ml}$ were collected in EDTA-2Natubes on the day before surgery (pre-surgery) and about 3 - 5 weeks after surgery (post-surgery). Pre-surgery blood samples were obtained from all the patients, whereas post-surgery blood samples were collected from the patients showing ctDNA in pre-surgery plasma. Within 2 hours after collection, peripheral blood samples were centrifuged twice at $3000 \mathrm{rpm}$ for $10 \mathrm{~min}$ at room temperature to separate the plasma, and stored at $-80^{\circ} \mathrm{C}$. For examination, plasma was defrosted and centrifuged at $12,000 \mathrm{rpm}$ for $10 \mathrm{~min}$ at room temperature. Then, ctDNA was extracted from 2 $\mathrm{ml}$ of plasma with QIAamp Circulating Nucleic Acid Kit (Qiagen) according to the manufacturer's instructions.

\subsection{Sanger Sequencing of Genomic DNA}

PCR primers used for amplifying fragments of the PIK3CA gene exon 9 and exon 20 and the nested sequencing primers used for Sanger sequencing are as follows: PIK3CA E542K and E545K; forward (5'-ttacagagtaacagactagc-3'), reverse 


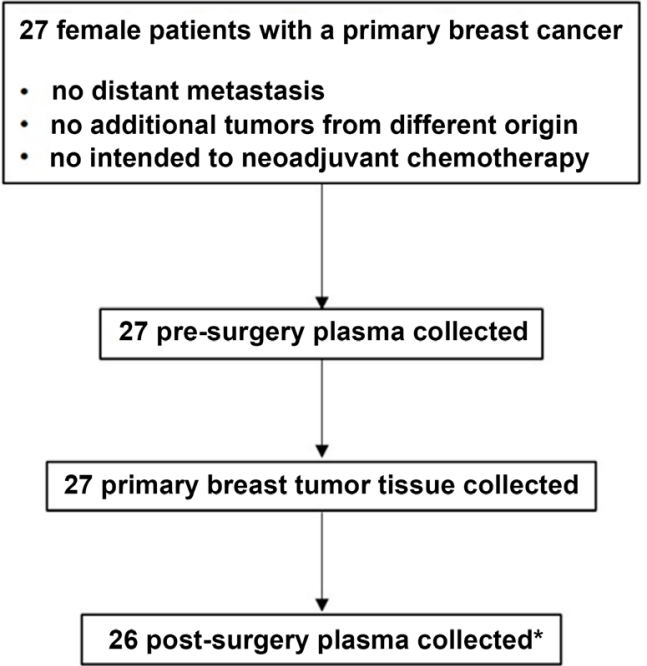

Figure 1. Enrollment of patients and sample collection. Twenty-seven female patients with a primary breast cancer were enrolled in this prospective study. Mutation of the PIK $3 C A$ gene was analyzed in genomic DNA from FFPE specimens from tumors and pre- and post-plasma samples. ${ }^{\star}$ Post-surgery plasma was not collected from one patient.

(5'-cttacctgtgactccatagaa $\left.-3^{\prime}\right)$ and sequencing (5'-gctagagacaatgaattaaggg $\left.-3^{\prime}\right)$, and PIK3 CA H1047R; forward (5'-gatgacattgcatacattcg - $3^{\prime}$ ), reverse (5'-gtggaagatccaatccattt $\left.-3^{\prime}\right)$, and sequencing ( $5^{\prime}$-cgaaagaccctagccttag $\left.-3^{\prime}\right)$, as described by Beaver, J. A. et al. [10].

\subsection{Droplet Digital PCR of Genomic DNA and ctDNA}

Droplet digital PCR (ddPCR) was used to detect PIK3CA mutation in genomic DNA from FFPE samples and ctDNA from plasma. Primers and probes were as follows; PrimePCR ddPCR Mutation Assay Kit: PIK3CA WT for p.E542K and PIK3CA p.E542K, Human (Bio-Rad Laboratories, Inc.\#1863131), PIK3CA WT for p.E545K and PIK3CA p.E545K, Human (Bio-Rad Laboratories, Inc. \#1863132), and PIK3CA WT for p.H1047R and PIK3CA p.H1047R, Human (Bio-Rad Laboratories, Inc. \#1863133). Briefly, DNA samples were mixed with primers, probes, and ddPCR ${ }^{\mathrm{mw}}$ Supermix for Probes (no dUTP) (Bio-Rad Laboratories, Inc. \#1863023) in total volume of $20 \mu$ l. About 20,000 droplets were made from the mixture and droplet-generating oil using QX200 droplet generator (Bio-Rad Laboratories, Inc.). Target genes were amplified in these droplets with a thermal cycler. PCR products were loaded onto the QX200 Droplet Reader (Bio-Rad Laboratories, Inc.) and the data were analyzed with Quanta Soft Software.

\subsection{Statistical Analysis}

Fisher's exact test was applied using GraphPad Prism V.5.04 (GraphPad Software, San Diego, CA) to analyze correlation between clinico-pathological fea- 
tures and $P I K 3 C A$ mutation in primary tumor or ctDNA.

\section{Results}

\subsection{Characteristics of Patients}

Characteristics of patients are shown in Table 1. The median age of the patients was 75 years-old, whereas the median period to be followed up the patients was 16 months. No clinical recurrence or metastasis was observed during the follow-up period in these patients. Twenty-seven primary breast tumors examined include 24 (89\%) invasive ductal carcinoma, two (7\%) invasive lobular carcinoma, and one (4\%) mucinous carcinoma. Sixteen tumors showed diameters of more than $2 \mathrm{~cm}$ (T2 and T3; 59\%), whereas 11 tumors showed diameters of less than $2 \mathrm{~cm}(\mathrm{~T} 1 ; 41 \%)$. Lymph node metastasis was detected in eight patients (N1: $30 \%)$. Hormonal state of tumors were determined by immunohistochemistry and 4 molecular subtypes of tumors were determined as follows; tumors with estrogen receptor(ER)-positive, progesterone receptor(PgR)-positive, human epidermal growth factor receptor 2(HER2)-negative, and Ki-67 $<20 \%$ as luminal A-like breast cancer; tumors with ER-positive and HER2-positive, or ER-positive, HER2-negative, and PgR-negative or Ki-67 > 20\% as luminal B-like breast cancer; tumors with ER-negative and HER2-positive as HER2-enriched breast cancer; tumors with ER-negative, PgR-negative, and HER2-negative as basal-like breast cancer. According to this classification, 12 of 27 tumors (44\%) examined were luminal A-like, 13 (48\%) were luminal B-like, and two (7\%) were HER2 type breast cancer.

\subsection{Analysis of PIK3CA Mutation in FFPE Specimens of Primary Breast Cancer}

Mutation of the PIK3CA gene was analyzed in genomic DNA from FFPE specimens of 27 primary breast cancer using Sanger sequencing and ddPCR (Figure 2 ). We adopted $1 \%$ of fractional abundance as the cut-off value. In total, the PIK3 CA mutations were detected in 13 of 27 tumors (48\%), including one tumor with E542K (8\%), four with E545K (30\%), seven with H1047R (54\%), and one with both E542K and H1047R (8\%) (Table 2). Fractional abundance of the mutated PIK3CA allele in these tumors ranged from $2.2 \%$ to $43.6 \%$. The PIK3 CA mutations were detected in 12 (48\%) of 25 ER-positive tumors; 5 (42\%) of 12luminal A-like tumors, 7 (54\%) of 13 luminal B-like tumors; and 1 (50\%) of 2 HER2-type tumors. No significant correlation was observed between the PIK3CA mutation in primary tumors and patients' age, histological type, $\mathrm{T}$ stage, nodal status, PgR status, HER2 status, Ki-67 index, and molecular subtypes (Table 3).

\subsection{Analysis of PIK3CA Mutation in Pre-Surgery ctDNA}

Next, the PIK3CA mutations in ctDNA was examined by ddPCR in matched pre-surgery plasma from 13 patients with tumors carrying the $P I K 3 C A$ 
Table 1. Clinico-pathological characteristics of the patients examined.

\begin{tabular}{|c|c|c|}
\hline Median age (years old) & & $75(31-85)$ \\
\hline Follow-up period (months) & & $16(6-21)$ \\
\hline \multicolumn{3}{|l|}{ Histological type } \\
\hline & ${ }^{*} \mathrm{IDC}$ & 24 \\
\hline & ${ }^{* *}$ ILC & 2 \\
\hline & special type & 1 \\
\hline \multicolumn{3}{|l|}{$\mathrm{T}$} \\
\hline & $\mathrm{T} 1$ & 11 \\
\hline & $\mathrm{T} 2$ & 14 \\
\hline & T3 & 2 \\
\hline \multicolumn{3}{|l|}{$\mathrm{N}$} \\
\hline & No & 19 \\
\hline & N1 & 8 \\
\hline \multicolumn{3}{|l|}{ ER } \\
\hline & positive & 25 \\
\hline & negative & 2 \\
\hline \multicolumn{3}{|l|}{ PgR } \\
\hline & positive & 18 \\
\hline & negative & 9 \\
\hline \multicolumn{3}{|l|}{ HER2 ${ }^{* * *}$} \\
\hline & positive & 5 \\
\hline & negative & 22 \\
\hline \multicolumn{3}{|l|}{ Ki-67 } \\
\hline & $<20 \%$ & 15 \\
\hline & $\geq 20 \%$ & 12 \\
\hline \multicolumn{3}{|l|}{ Molecular Subtype } \\
\hline & luminal A-like & 9 \\
\hline & luminal B-like & 16 \\
\hline & HER2-enriched & 2 \\
\hline & Basal-like & 0 \\
\hline
\end{tabular}

${ }^{*}$ IDC: Invasive Ductal Carcinoma; ${ }^{* *}$ ILC: Invasive Lobular Carcinoma; ${ }^{* *}$ HER2-: IHC 0, 1+ or 2+ (FISH-); HER2+: IHC $3+$ or $2+$ (FISH+).

mutations in their primary tumors (Table 2). Fractional abundance of $0.1 \%$ was defined as the cut-off value. Four of 13 pre-surgery ctDNA (31\%) showed the same mutations as those in primary tumors, including 1 mutation at E545K and 


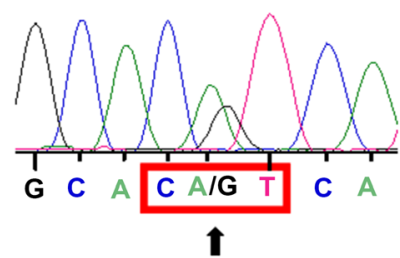

c. $3140 A$ > G; H1047R

(a)

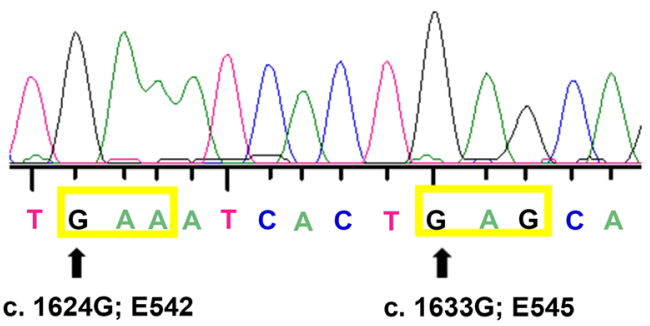

(c)

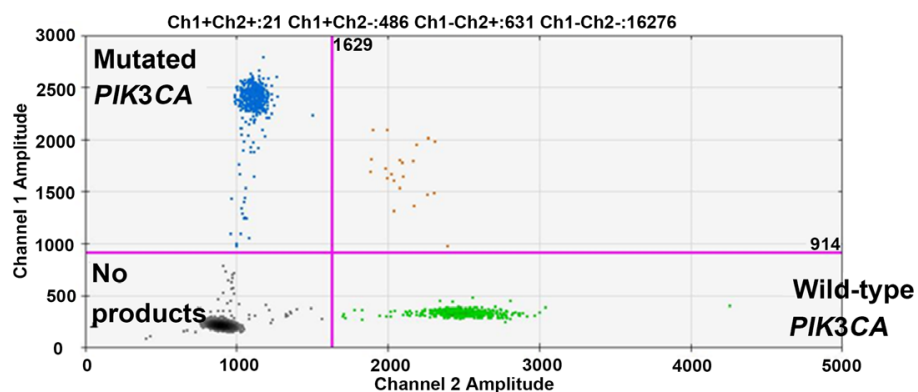

(b)

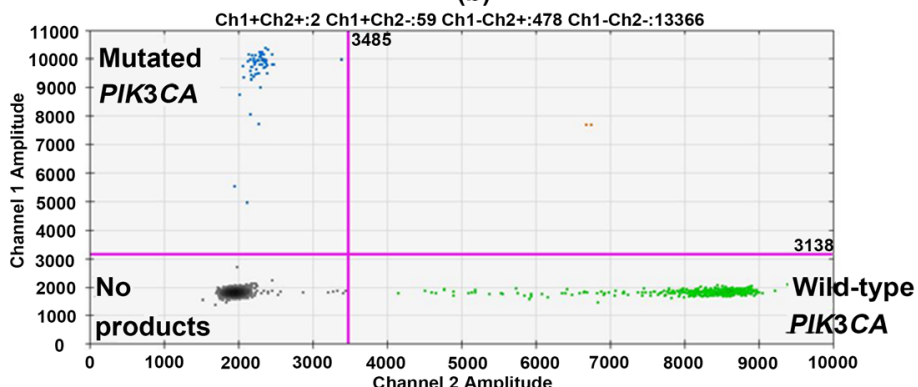

(d)

Figure 2. Detection of the PIK3CA mutation in primary tumor DNA from FFPE specimens. The results were shown in case No. 6 with the PIK3CA mutation at H1047R by Sanger sequencing (a) or ddPCR (b), and in case No. 2 with the PIK3CA mutation at E545K by Sanger sequencing (c) or ddPCR (d).

3 mutations at H1047R. Fractional abundance of the mutated PIK3CA allele in pre-surgery ctDNA was ranged from $0.20 \%$ to $1.08 \%$ and did not show significant correlation with fractional abundance in primary tumors. Four tumors with the mutated $P I K 3 C A$ in pre-surgery ctDNA include 3 invasive ductal carcinoma (IDC) with luminal B-like (2 cases) or HER2-enriched (1 case) subtypes and 1 invasive lobular carcinoma (ILC) with luminal A-like subtype. Two of 4 tumors showed lymphnode metastasis in stages IIB and IIIA, whereas the other 2 tumors were in stage IA without nodal involvement. However, no significant correlation between the PIK3CA mutation in pre-surgery ctDNA and clinico-pathological features of primary tumors (Table 3 ).

\subsection{Analysis of the PIK3CA Mutation in Post-Surgery ctDNA}

Finally, the PIK3CA mutation was examined in matched post-surgery ctDNA in plasma from 12 of 13 patients with tumors harboring the PIK3CA mutations, and the same mutations as those in primary tumors were detected in $2(16.6 \%)$ of 12 post-surgery ctDNA (Table 2). As expected, these 2 cases (cases 7 and 8 ) also carried the mutated $P I K 3 C A$ in pre-surgery ctDNA. However, no mutated $P I K 3 C A$ was detected in the other 2 cases (cases 5 and 6). The fractional abundance of the mutated $P I K 3 C A$ in pre-surgery ctDNA was $0.96 \%$ in case 7 and $1.08 \%$ in case 8 , which was higher than that in case $5(0.20 \%)$ or case $6(0.54 \%)$.

\section{Discussion}

In the present study, we developed a system to detect the PIK3CA mutation in ctDNA in 27 cases of Japanese breast cancer in early-stage and examined their 
Table 2. Analysis of the PIK3CA mutation in tumors and plasma.

\begin{tabular}{|c|c|c|c|c|c|c|c|c|c|c|c|c|c|c|c|}
\hline & & & & & & & & & & \multicolumn{2}{|c|}{ Primary tumor } & \multicolumn{2}{|c|}{ Pre-surgery ctDNA } & \multicolumn{2}{|c|}{ Post-surgery ctDNA } \\
\hline $\begin{array}{l}\text { Case } \\
\text { No. }\end{array}$ & Age & $\begin{array}{c}\text { Histological } \\
\text { type }^{\star}\end{array}$ & $\mathrm{TNM}^{* *}$ & Stage & ER & PgR & $\mathrm{HER} 2^{* * *}$ & $\mathrm{Ki}-67$ & $\begin{array}{l}\text { Molecular } \\
\text { subtype }\end{array}$ & PIK3CA & $\begin{array}{l}\text { Fractional } \\
\text { abundance }\end{array}$ & PIK3 $3 A$ & $\begin{array}{l}\text { Fractional } \\
\text { abundance }\end{array}$ & PIK $3 C A$ & $\begin{array}{l}\text { Fractional } \\
\text { abundance }\end{array}$ \\
\hline 1 & 55 & IDC & T2N0 & IIA & positive & negative & positive & $25 \%$ & luminal B & E542K & $1.6 \%$ & wild-type & & wild-type & \\
\hline 2 & 76 & ILC & $\mathrm{T} 3 \mathrm{~N} 1$ & IIIA & positive & negative & negative & $<10 \%$ & luminal B & E545K & $11.0 \%$ & wild-type & & wild-type & \\
\hline 3 & 51 & IDC & T2N0 & IIA & positive & positive & negative & $12 \%$ & luminal A & E545K & $22.6 \%$ & wild-type & & wild-type & \\
\hline 4 & 79 & IDC & T2N0 & IIA & positive & negative & negative & $35 \%$ & luminal B & E545K & $16.2 \%$ & wild-type & & wild-type & \\
\hline 5 & 78 & IDC & $\mathrm{T} 2 \mathrm{~N} 1$ & IIB & positive & negative & negative & $6 \%$ & luminal B & E545K & $13.1 \%$ & E545K & $0.20 \%$ & wild-type & \\
\hline 6 & 75 & IDC & T1cN0 & IA & positive & positive & positive & $>50 \%$ & luminal B & H1047R & $43.6 \%$ & H1047R & $0.54 \%$ & wild-type & \\
\hline 7 & 47 & ILC & T3N1 & IIIA & positive & positive & negative & $15 \%$ & luminal A & H1047R & $31.0 \%$ & H1047R & $0.96 \%$ & H1047R & $0.30 \%$ \\
\hline 8 & 65 & IDC & T1bN0 & IA & negative & negative & positive & $9 \%$ & HER2 & H1047R & $10.2 \%$ & H1047R & $1.08 \%$ & H1047R & $0.27 \%$ \\
\hline 9 & 85 & IDC & T1cN0 & IA & positive & positive & negative & $22 \%$ & luminal B & H1047R & $32.5 \%$ & wild-type & & wild-type & \\
\hline 10 & 79 & IDC & $\mathrm{T} 2 \mathrm{~N} 1$ & IIB & positive & positive & negative & $50 \%$ & luminal B & H1047R & $2.2 \%$ & wild-type & & wild-type & \\
\hline 11 & 75 & IDC & T2N0 & IIA & positive & positive & positive & $50 \%$ & luminal B & H1047R & $26.9 \%$ & wild-type & & wild-type & \\
\hline 12 & 84 & IDC & T1cN0 & IA & positive & positive & negative & $6 \%$ & luminal A & E542K & $3.8 \%$ & wild-type & & wild-type & \\
\hline & & & & & & & & & & H1047R & $22.5 \%$ & wild-type & & wild-type & \\
\hline 13 & 39 & IDC & T1cN0 & IA & positive & positive & negative & $12 \%$ & luminal $\mathrm{A}$ & H1047R & $5.3 \%$ & wild-type & & $\begin{array}{c}\text { not } \\
\text { collected }\end{array}$ & \\
\hline 14 & 79 & IDC & T1cN0 & IA & positive & positive & negative & $16 \%$ & luminal A & wild-type & & & & & \\
\hline 15 & 56 & IDC & $\mathrm{T} 1 \mathrm{cN} 1$ & IIA & positive & positive & negative & $22 \%$ & luminal B & wild-type & & & & & \\
\hline 16 & 81 & IDC & T2N0 & IIA & negative & negative & positive & $>50 \%$ & HER2 & wild-type & & & & & \\
\hline 18 & 31 & IDC & T2N0 & IIA & positive & positive & negative & $12 \%$ & luminal $\mathrm{A}$ & wild-type & & & & & \\
\hline 19 & 77 & IDC & T1cN0 & IA & positive & positive & negative & $<10 \%$ & luminal A & wild-type & & & & & \\
\hline 20 & 47 & IDC & T1aN0 & IA & positive & positive & negative & $10 \%$ & luminal A & wild-type & & & & & \\
\hline 21 & 63 & IDC & $\mathrm{T} 2 \mathrm{~N} 1$ & IIB & positive & negative & negative & $13 \%$ & luminal B & wild-type & & & & & \\
\hline 22 & 51 & IDC & T2N0 & IIA & positive & positive & negative & $25 \%$ & luminal B & wild-type & & & & & \\
\hline 23 & 80 & IDC & $\mathrm{T} 2 \mathrm{~N} 1$ & IIB & positive & positive & negative & $25 \%$ & luminal B & wild-type & & & & & \\
\hline 24 & 83 & special type & T2N0 & IIA & positive & negative & negative & $12 \%$ & luminal B & wild-type & & & & & \\
\hline 25 & 59 & IDC & $\mathrm{T} 1 \mathrm{cN} 1$ & IIA & positive & negative & negative & $8 \%$ & luminal B & wild-type & & & & & \\
\hline 26 & 44 & IDC & T2N0 & IIA & positive & positive & negative & $22 \%$ & luminal B & wild-type & & & & & \\
\hline 27 & 75 & IDC & T2No & IIA & positive & positive & negative & $22 \%$ & luminal B & wild-type & & & & & \\
\hline
\end{tabular}

${ }^{\star}$ IDC, invasive ductal carcinoma; ILC, invasive lobular carcinoma. ${ }^{* *}$ All cases were in M0. ${ }^{* *}$ HER2 negative, IHC $0,1+$ or $2+($ FISH-); HER2 positive: IHC $3+$ or $2+$ $(\mathrm{FISH}+)$.

clinico-pathological significance. Three common mutations of the PIK $3 C A$ gene were selected as targets of tumor-specific DNA alterations that were sensitively detectable by ddPCR due to their high incidence of mutation in breast cancer. At the beginning, the PIK $3 C A$ mutations were detected in $48 \%$ of luminal subtype and $50 \%$ of HER2-enriched subtype of primary breast cancer in this study, which was consistent with the previous finding by Network et al. [6]. Absence of 
Table 3. Clinico-pathological characteristics of the cases with the PIK $3 C A$ mutation in primary tumors or pre-surgery ctDNA.

\begin{tabular}{|c|c|c|c|c|c|c|}
\hline & prim & $=27)$ & & pre-ct & $1=13$ & \\
\hline & & & & & & \\
\hline & WT & mut & $P$ & WT & mut & $P$ \\
\hline$<75$ y.o. & 7 & 5 & ns\# & 3 & 2 & ns \\
\hline$>75$ y.o. & 7 & 8 & & 6 & 2 & \\
\hline IDC $^{*}$ & 13 & 11 & ns & 8 & 3 & ns \\
\hline $\operatorname{ILC}^{\star *}$ & 1 & 1 & & 1 & 1 & \\
\hline $\mathrm{T} 1$ & 6 & 5 & ns & 3 & 2 & ns \\
\hline $\mathrm{T} 2-\mathrm{T} 3$ & 8 & 8 & & 6 & 2 & \\
\hline No & 10 & 9 & ns & 7 & 2 & ns \\
\hline N1 & 4 & 4 & & 2 & 2 & \\
\hline PgR+ & 10 & 8 & ns & 6 & 2 & ns \\
\hline PgR- & 4 & 5 & & 3 & 2 & \\
\hline $\mathrm{Ki}-67<20 \%$ & 8 & 7 & ns & 4 & 3 & ns \\
\hline $\mathrm{Ki}-67>20 \%$ & 6 & 6 & & 5 & 1 & \\
\hline HER2- ${ }^{* * *}$ & 13 & 9 & ns & 7 & 2 & ns \\
\hline HER $2+^{* * *}$ & 1 & 4 & & 2 & 2 & \\
\hline luminal A-like & 7 & 5 & ns & 3 & 1 & $\mathrm{~ns}$ \\
\hline luminal B-like & 6 & 7 & & 6 & 2 & \\
\hline luminal type & 13 & 12 & ns & 9 & 3 & ns \\
\hline HER2-enriched type & 1 & 1 & & 0 & 1 & \\
\hline
\end{tabular}

\#ns: not significant difference. ${ }^{\star}$ IDC: Invasive Ductal Carcinoma. ${ }^{*}$ ILC: Invasive Lobular Carcinoma. ${ }^{* * *}$ HER2-: IHC $0,1+$ or $2+($ FISH-); HER $2+$ IHC $3+$ or $2+($ FISH+).

significant correlation between the PIK3CA mutation status in primary tumors and the clinico-pathological features demonstrated by Campbell et al. [14] was also confirmed in this study.

As to the early-stage of breast cancer, Beaver et al. analyzed two common PIK $3 C A$ mutations at E545K and H1047R in ctDNA from pre- and post-surgery plasma, as well as primary tumors, from 29 patients with early-stage breast can- 
cer using ddPCR. They subsequently detected the mutant PIK3CA in 13 of 14 (93\%) cases of ctDNA from pre-surgery plasma of the patients harboring the relevant mutation in primary tumors [10]. The higher detection rate of mutation in pre-surgery ctDNA in their study in comparison with that of 33\% in our study would be caused by their unique procedure of digital PCR platform, including 22-cycles of pre-amplification of ctDNA and the low cut-off value of $0.01 \%$. It is noteworthy that $85 \%$ of the fractional abundance of the mutated PIK3CA in ctDNA is lower than $0.1 \%$ even after pre-amplification in their study. In our study, by contrast, we directly quantified the ctDNA without pre-amplification by ddPCR and adopted the cut-off value of $0.1 \%$, ten-fold higher than that in the study by Beaver et al. [10], considering the difference in the detection rate of mutation in the platform by RainDance Technologies (Billerica, MA, USA) in their analysis and in that by Bio-Rad Laboratories (Hercules, CA, USA) in our analysis. It is also interesting that the detection rate of pre-surgery ctDNA in early breast cancer was reported to be $23 \%$ in another retrospective study using serum as a source of ctDNA [11], suggesting that serum would be less appropriate for this type of analysis.

In this study, we identified 4 cases (case Nos. 5, 6, 7, and 8) with the mutated $P I K 3 C A$ in ctDNA from pre-surgery plasma out of 13 cases carrying the mutated PIK $3 C A$ in primary tumors. These 4 cases, however, did not show any specific features correlated with clinico-pathological findings of the tumors, suggesting that the presence of ctDNA could be an independent factor from known pathological features of the primary tumors or prognostic factors such as nodal involvement or Ki-67 index. In fact, two of the 4 cases (Case Nos. 6 and 8) were from breast cancer in stage IA, a very early-stage without nodal involvement. More importantly, 2 of the 4 cases (Case Nos. 7 and 8) gave the identical signals of the mutated PIK3CA in ctDNA from post-surgery plasma as well. Considering the short half-life of ctDNA, post-surgery ctDNA in cases No. 7 and No. 8 would be originated from residual disease not detected in macroscopic examination or imaging analyses. In fact, it was reported that the overall survival rate and recurrence-free survival rate were significantly lower in patients carrying ctDNA [11]. It is interesting that the fractional abundance of the mutated PIK3CA in cases $7(0.96 \%)$ and $8(1.08 \%)$ were higher than that in cases $5(0.20 \%)$ and 6 $(0.54 \%)$ as shown in Table 2 . If higher fractional abundance in pre-surgery ctDNA might be caused by higher tumor burden at the time of surgical operation, ctDNA could provide a possible indicator of the residual disease after surgery and so possible recurrence or poor prognosis of the patients, although the cases 7 and 8, as well as all other cases, are being followed up at present without any clinical recurrence or metastasis and their final outcome has not been determined yet.

In this connection, it should be noted that the risk of recurrence in case No. 8 is estimated to be low and adjuvant chemotherapy would not be recommended on the basis of conventional therapeutic guideline. However, if ctDNA in post-surgery plasma could be originated from the residual disease, including regional and/or 
metastatic breast cancer, additional systemic chemotherapy after surgical operation would be definitely required for case No. 8 to prevent incurable recurrence of breast cancer and to improve prognosis of the patients. On the other hand, conventional guideline would strongly recommend case No. 5 to receive adjuvant chemotherapy, although ctDNA detected in pre-surgery plasma disappeared in post-surgery ctDNA in this study. Similarly, current guideline would recommend cases No. 2 and No. 10 with nodal involvement to receive adjuvant chemotherapy, although ctDNA was not detected even in pre-surgery plasma. In these cases, however, surgical treatment would be effective enough to gain complete cure, whereas additional adjuvant chemotherapy might become overtreatment for the patients.

One of the limitations in this study is a small number of cases examined. In addition, the final outcome of breast cancer in individual cases has not been determined yet in this prospective study. A larger number of breast cancers in early-stage with significant information of the patients' outcome would be required to draw a definite conclusion. Another limitation is the incidence of mutation in the target genes, because only cases carrying the mutated targets in primary tumors are informative in the analysis of presence or absence of ctDNA. In spite of these limitations, however, we could detect PIK $3 C A$ mutation in $53 \%$ of primary breast cancers using ddPCR. Since the system of mutation-detection by ddPCR is only applicable to a single point mutation, 3 common mutations of the PIK $3 C A$ would provide the most potent target of ctDNA in breast cancer.

\section{Conclusion}

In conclusion, we developed a system to detect ctDNA in the plasma from breast cancer patients by ddPCR using the mutated PIK $3 C A$ as targets. ctDNA carrying the mutated PIK $3 C A$ was detected in pre-surgery plasma in $33 \%$ of informative cases of breast cancer in early-stage. ctDNA was further detected in a subset of cases at 3 - 5 weeks after surgery, suggesting the presence of residual disease. The fractional abundance of the mutated PIK3CA in pre-surgery ctDNA could provide a possible indicator to predict the residual disease of breast cancer after surgery and to choose the appropriate candidate for adjuvant chemotherapy.

\section{References}

[1] Diaz, L.A. and Bardelli, A. (2014) Liquid Biopsies: Genotyping Circulating Tumor DNA. Journal of Clinical Oncology, 32, 579-586. https://doi.org/10.1200/JCO.2012.45.2011

[2] Murtaza, M., Dawson, S.J., Tsui, D.W., Gale, D., Forshew, T., Piskorz, A.M., Parkinson, C., Chin, S.F., Kingsbury, Z., Wong, A.S., Marass, F., Humphray, S., Hadfield, J., Bentley, D., Chin, T.M., Brenton, J.D., Caldas, C. and Rosenfeld, N. (2013) Non-Invasive Analysis of Acquired Resistance to Cancer Therapy by Sequencing of Plasma DNA. Nature, 497, 108-112. https://doi.org/10.1038/nature12065

[3] Diehl, F., Schmidt, K., Choti, M.A., Romans, K., Goodman, S., Li, M., Thornton, K., Agrawal, N., Sokoll, L., Szabo, S.A., Kinzler, K.W., Vogelstein, B. and Diaz, L.A. 
(2008) Circulating Mutant DNA to Assess Tumor Dynamics. Nature Medicine, 14, 985-990. https://doi.org/10.1038/nm.1789

[4] Haber, D.A. and Velculescu, V.E. (2014) Blood-Based Analyses of Cancer: Circulating Tumor Cells and Circulating Tumor DNA. Cancer Discovery, 4, 650-661. https://doi.org/10.1158/2159-8290.CD-13-1014

[5] Hindson, C.M., Chevillet, J.R., Briggs, H.A., Gallichotte, E.N., Ruf, I.K., Hindson, B.J., Vessella, R.L. and Tewari, M. (2013) Absolute Quantification by Droplet Digital PCR versus Analog Real-Time PCR. Nature Methods, 10, 1003-1005. https://doi.org/10.1038/nmeth.2633

[6] Network, C.G.A. (2012) Comprehensive Molecular Portraits of Human Breast Tumours. Nature, 490, 61-70. https://doi.org/10.1038/nature11412

[7] Karakas, B., Bachman, K.E. and Park, B.H. (2006) Mutation of the PIK3CA Oncogene in Human Cancers. British Journal of Cancer, 94, 455-459. https://doi.org/10.1038/sj.bjc.6602970

[8] Higgins, M.J., Jelovac, D., Barnathan, E., Blair, B., Slater, S., Powers, P., Zorzi, J., Jeter, S.C., Oliver, G.R., Fetting, J., Emens, L., Riley, C., Stearns, V., Diehl, F., Angenendt, P., Huang, P., Cope, L., Argani, P., Murphy, K.M., Bachman, K.E., Greshock, J., Wolff, A.C. and Park, B.H. (2012) Detection of Tumor PIK3CA Status in Metastatic Breast Cancer Using Peripheral Blood. Clinical Cancer Research, 18, 3462-3469. https://doi.org/10.1158/1078-0432.CCR-11-2696

[9] Dawson, S.J., Tsui, D.W., Murtaza, M., Biggs, H., Rueda, O.M., Chin, S.F., Dunning, M.J., Gale, D., Forshew, T., Mahler-Araujo, B., Rajan, S., Humphray, S., Becq, J., Halsall, D., Wallis, M., Bentley, D., Caldas, C. and Rosenfeld, N. (2013) Analysis of Circulating Tumor DNA to Monitor Metastatic Breast Cancer. New England Journal of Medicine, 368, 1199-1209. https://doi.org/10.1056/NEJMoa1213261

[10] Beaver, J.A., Jelovac, D., Balukrishna, S., Cochran, R.L., Croessmann, S., Zabransky, D.J., Wong, H.Y., Valda Toro, P., Cidado, J., Blair, B.G., Chu, D., Burns, T., Higgins, M.J., Stearns, V., Jacobs, L., Habibi, M., Lange, J., Hurley, P.J., Lauring, J., Van Den Berg, D.A., Kessler, J., Jeter, S., Samuels, M.L., Maar, D., Cope, L., Cimino-Mathews, A., Argani, P., Wolff, A.C. and Park, B.H. (2014) Detection of Cancer DNA in Plasma of Patients with Early-Stage Breast Cancer. Clinical Cancer Research, 20, 2643-2650. https://doi.org/10.1158/1078-0432.CCR-13-2933

[11] Oshiro, C., Kagara, N., Naoi, Y., Shimoda, M., Shimomura, A., Maruyama, N., Shimazu, K., Kim, S.J. and Noguchi, S. (2015) PIK3CA Mutations in Serum DNA Are Predictive of Recurrence in Primary Breast Cancer Patients. Breast Cancer Research and Treatment, 150, 299-307. https://doi.org/10.1007/s10549-015-3322-6

[12] Takeshita, T., Yamamoto, Y., Yamamoto-Ibusuki, M., Inao, T., Sueta, A., Fujiwara, S., Omoto, Y. and Iwase, H. (2015) Prognostic Role of PIK3CA Mutations of Cell-Free DNA in Early-Stage Triple Negative Breast Cancer. Cancer Science, 106, 1582-1589. https://doi.org/10.1111/cas.12813

[13] Bettegowda, C., Sausen, M., Leary, R.J., Kinde, I., Wang, Y., Agrawal, N., Bartlett, B.R., Wang, H., Luber, B., Alani, R.M., Antonarakis, E.S., Azad, N.S., Bardelli, A., Brem, H., Cameron, J.L., Lee, C.C., Fecher, L.A., Gallia, G.L., Gibbs, P., Le, D., Giuntoli, R.L., Goggins, M., Hogarty, M.D., Holdhoff, M., Hong, S.M., Jiao, Y., Juhl, H.H., Kim, J.J., Siravegna, G., Laheru, D.A., Lauricella, C., Lim, M., Lipson, E.J., Marie, S.K., Netto, G.J., Oliner, K.S., Olivi, A., Olsson, L., Riggins, G.J., Sartore-Bianchi, A., Schmidt, K., Shih, L., Oba-Shinjo, S.M., Siena, S., Theodorescu, D., Tie, J., Harkins, T.T., Veronese, S., Wang, T.L., Weingart, J.D., Wolfgang, C.L., Wood, L.D., Xing, D., Hruban, R.H., Wu, J., Allen, P.J., Schmidt, C.M., Choti, M.A., Velculescu, V.E., Kinzler, K.W., Vogelstein, B., Papadopoulos, N. and Diaz, L.A. 
(2014) Detection of Circulating Tumor DNA in Early- and Late-Stage Human Malignancies. Science Translational Medicine, 6, $224 \mathrm{ra} 24$.

https://doi.org/10.1126/scitranslmed.3007094

[14] Campbell, I.G., Russell, S.E., Choong, D.Y., Montgomery, K.G., Ciavarella, M.L., Hooi, C.S., Cristiano, B.E., Pearson, R.B. and Phillips, W.A. (2004) Mutation of the PIK3CA Gene in Ovarian and Breast Cancer. Cancer Research, 64, 7678-7681.

https://doi.org/10.1158/0008-5472.CAN-04-2933 